\title{
AN ELECTRON TUBE AMPLIFIER USING 60-CYCLE ALTERNATING CURRENT TO SUPPLY POWER FOR THE FILAMENTS AND PLATES.
}

\author{
By P. D. Lowell.
}

ABSTRACT.

Electron tube amplifiers now form an important part of practically all radio receiving sets except the most simple types. Storage cells, which have heretofore usually been required for operating amplifiers, require constant attention, are bulky and heavy, and have other serious disadvantages. The dry cells, which are often used as a source of plate voltage, have a comparatively short life and are expensive and inconvenient. It would be much more convenient to use an amplifier which could be supplied with power from rro-volt, 60-cycle mains for both filaments and plates.

This paper describes a five-stage amplifier, which operates satisfactorily on 6ocycle supply, for both filaments and plates. This amplifier has three radio-frequency stages and two audio-frequency stages, and uses a crystal detector. A special transformer with five windings is used, the primary being supplied with r ro volts alternating current. The 60 -cycle current, when used in an ordinary amplifier circuit, introduces a strong 6o-cycle note which offers serious interference. This has been practically eliminated by balancing resistances, grid condensers, and special grid leaks of comparatively low resistance, telephone transformer in the output circuit, and crystal detector instead of electron tube detector. In the final form of the amplifier there is only a slight residual hum, which is not objectionable. The amplification obtained with alternating-current supply was as good as that obtained with the same amplifier used with-direct-current supply. The complete unit is light, compact, and portable. For the reception of damped waves, the amplifier as constructed operated most satisfactorily for wave lengths from 200 to $75^{\circ}$ meters. This range was determined by the working range of the radio-frequency transformers used. By using suitable radio-frequency transformers, it is expected that the amplifier will be effective for the reception of damped waves and undamped waves as long as ro 000 meters. For the reception of undamped waves, a separate heterodyne should be employed. The paper gives circuit diagrams, and states the values of the condensers, resistors, and inductors used. The paper also describes a method for rectifying the rro-volt alternating-current supply and using it for the field of a loud speaker.

Electron tube amplifiers form an important part of practically all radio receiving sets now, except the most simple types. Such amplifiers are, in fact, necessary to receive distant stations, or when using coil antennas. For good operation, amplifier tubes require for the filament a source of voltage of very constant value (usually about 6 volts), and for the plate a source of voltage of from 40 to perhaps 300 volts. The filament voltage is usually supplied by storage cells and the plate voltage by dry cells. The 
maintenance of these cells in operating condition, especially the storage cells, is often a source of much difficulty and annoyance. The storage cells are necessarily bulky and heavy, require constant attention to maintain proper charge and density of electrolyte, give off injurious acid or other fumes, and are subject to variations of voltage during the period of discharge. The development of an amplifier which can be supplied from the ordinary r ro-volt alternating-current lighting mains is of considerable practical importance, since it would eliminate the practical difficulties of maintaining storage cells. The amplifier using such alternatingcurrent supply has the important advantages of reliability, convenience, and cheapness both in first cost and operating cost.

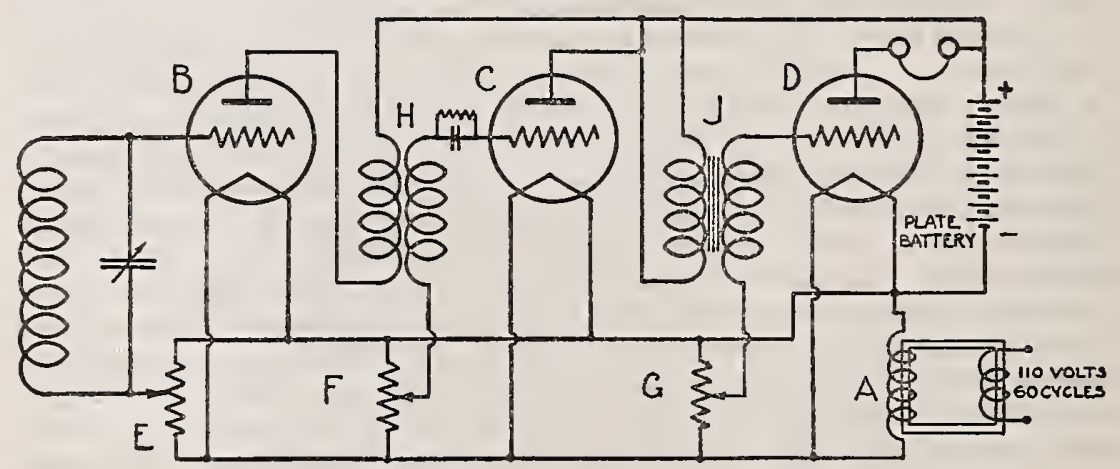

FIG. I.-Two-stage amplifier circuit using tube detector.

With alternating-current supply the expense of the storage battery and its charging equipment and the expense of the dry battery for the plate are eliminated. The first cost of the amplifier with alternating-current supply is somewhat less than the first cost of the ordinary amplifier, including its necessary batteries and charging equipment. One of the most important items in the maintenance cost of the ordinary amplifier is the dry cells for the " $B$ " battery, since in service these dry cells must be renewed about every three to six months. It is estimated that during a period of five years the saving in the cost of " $\mathrm{B}$ " dry batteries will alone pay for a complete amplifier with alternating-current supply.

An amplifier with alternating-current supply, using five stages of amplification, has been developed at the Bureau of Standards. There are three stages of radio-frequency amplification and two stages of audio-frequency amplification. This paper describes this amplifier briefly.

The first arrangement tried consisted of one radio-frequency stage of amplification, tube detector, and one audio-frequency 
stage. The filaments of the three tubes were lighted by 6 volts supplied by a step-down transformer, the primary of which was connected to the rro-volt, 6o-cycle power mains. The circuit is shown in Figure I.

In Figure I, the filament lighting transformer is shown at $A$, supplying voltage for the filaments of electron tubes $B, C$, and $D$. The amplifier input circuit is connected to the grid of tube $B$ and to the slider of the balancing resistance $E$, the latter having a resistance of $200 \mathrm{ohms}$ and being connected across the filament line. A radio-frequency transformer is shown at $H$, a balancing resistance at $F$, an audio-frequency transformer at $J$, and another balancing resistance at $G$. The purpose of the balancing resistances $E, F, G$, is to keep the normal voltage of the grids at a steady value with respect to the average voltage of the

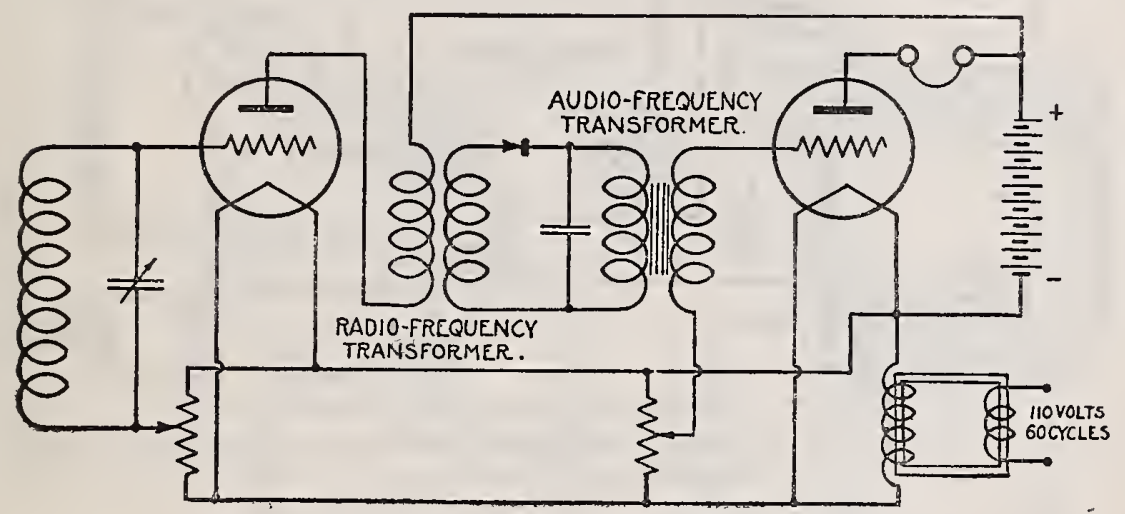

FIG. 2.-Two-stage amplifier using crystal deiector.

electrical midpoint of the filament, and to thus eliminate the hum which variations of the grid voltage would cause. These balancing resistances are adjusted until the hum is eliminated. Reception was accomplished with this circuit, but there was considerable 6o-cycle hum present in spite of the beneficial effect of the balancing resistances.

The employment of a crystal detector in place of the electron tube detector reduced the 6o-cycle hum very considerably. This circuit is shown in Figure 2.

When an electron tube is used as the detector, there is impressed on both the plate and the filament a 6o-cycle alternating-current voltage which, although small, becomes very objectionable when amplified by one or two stages of audio-frequency amplification. When the crystal detector is used, no 6o-cycle voltage is supplied $719^{\circ}-22-2$ 
to the detector circuit. The radio-frequency transformer, whose output is delivered to the detector circuit, prevents the passage in any appreciable amount of 6o-cycle current supplied to the radio-frequency stage, and such voltages are not present in the crystal detector circuit and do not reach the input of the audiofrequency stage.

The employment of a crystal detector may at first seem objectionable, due to the fact that as ordinarily used it is rather difficult to find a point of good sensitivity. But tests on this amplifier showed that careful adjustment of the crystal detector was not necessary because of the fact that the radio-frequency amplification preceding the detector usually gave sufficient signal strength so that a point of sufficient sensitivity could be easily found.

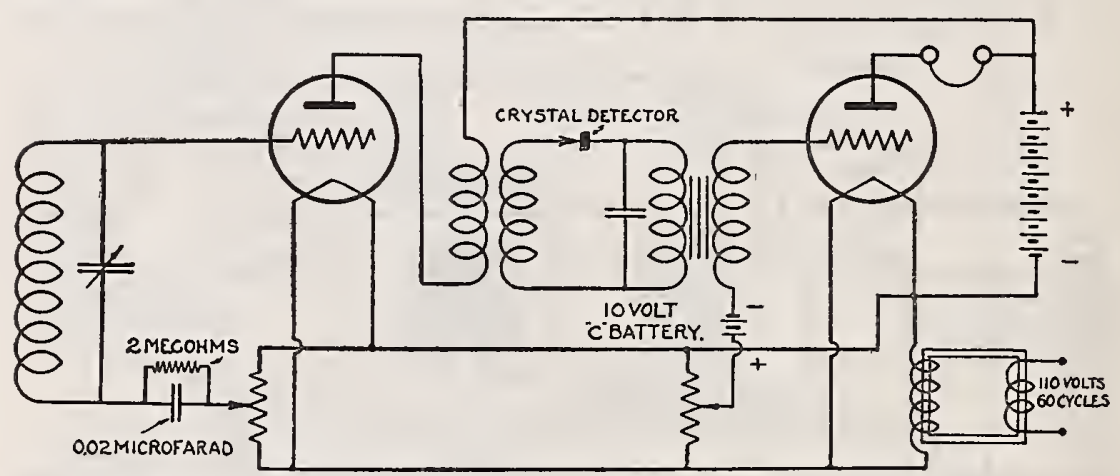

FIG. 3.-Two-stage amplifier using crystal detector, with grid condenser for radio stages and grid battery for audio stages.

This circuit gave quite good results. The 6o-cycle hurn was practically eliminated and the crystal detector gave almost as good rectification as the tube detector.

It was found that better amplifying action could be obtained by inserting condensers of about 0.02 microfarad capacity, shunted by two megohm grid-leak resistances, in the grid circuits, in series with the sliders of the balancing resistances. The grid condensers and grid-leak resistances allow the grids to assume a normal voltage which is more favorable for amplifying purposes. The leak resistances allow any accumulated charge on the grids to leak off to the filaments. Still better amplification and quieter operation was produced by replacing the series grid condenser and leak in the audio stage with a ro-volt battery giving a negative charge to the grid. A battery of dry cells was used for this purpose; since only an extremely small current is required, the life 
of the dry cells is practically their shelf life. This gave a circuit as shown in Figure 3.

Alternating current rectified by means of a gas-filled two-element rectifier tube ("Tungar" tube) was tried as a source of filament power, but the residual hum was much greater than when unrectified alternating current was used. This is because of the fact that during rectification the wave form becomes distorted and it becomes impossible to stabilize the grid voltage by means of the balancing resistances.

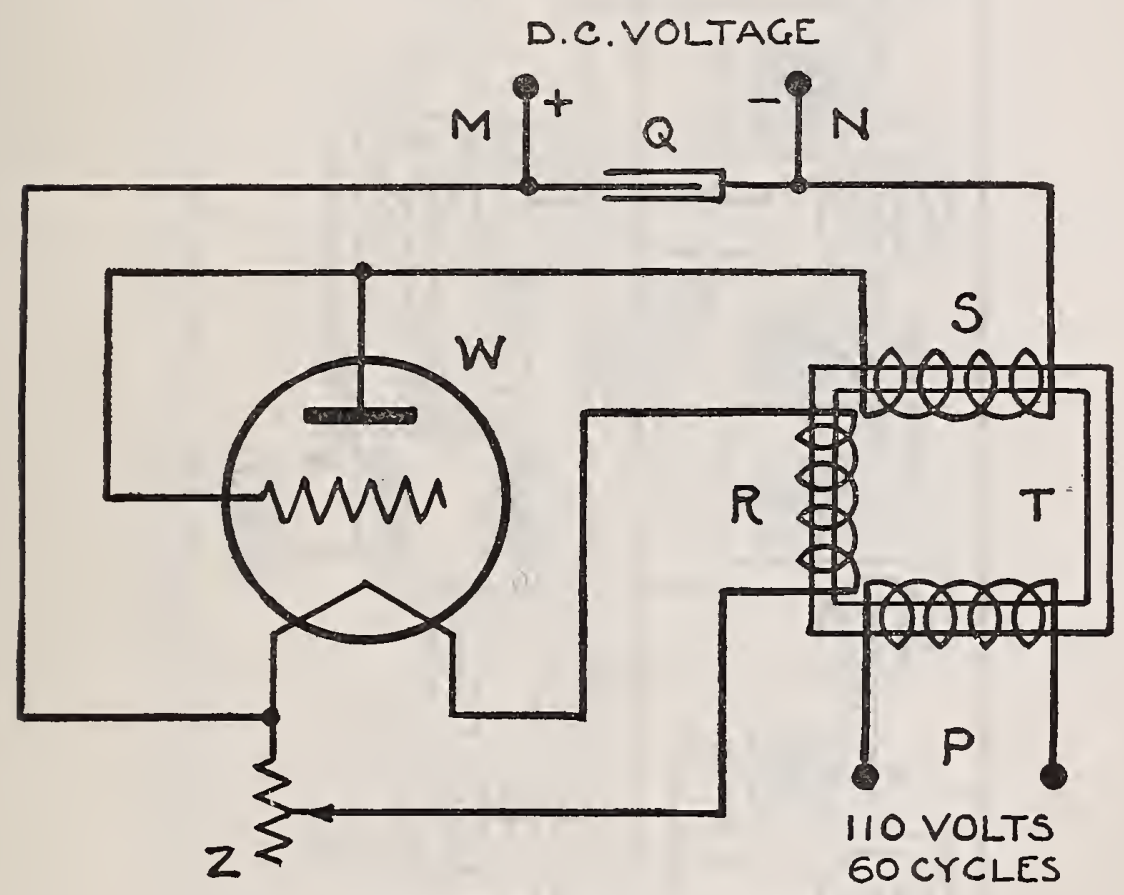

FIG. 4.-Rectification circuit for plate voltage supply.

In the above-mentioned tests, a plate battery was used for convenience, but this was replaced by alternating current which had been rectified by means of an electron tube and smoothed out with condensers of large capacity. The rectification circuit for the plate voltage supply is shown in Figure 4.

In Figure 4 , the primary $P$ of the transformer $T$ is connected to the power mains, and the winding $R$ gives 8 volts for the filament of the rectifier tube $W$. Winding $S$ gives 300 volts which is rectified by the tube $W$ and smoothed out by the condenser $Q$ which has about ro microfarads capacity. This gives at terminals $M$ and $N$ a high-voltage direct current which is quite suitable for 


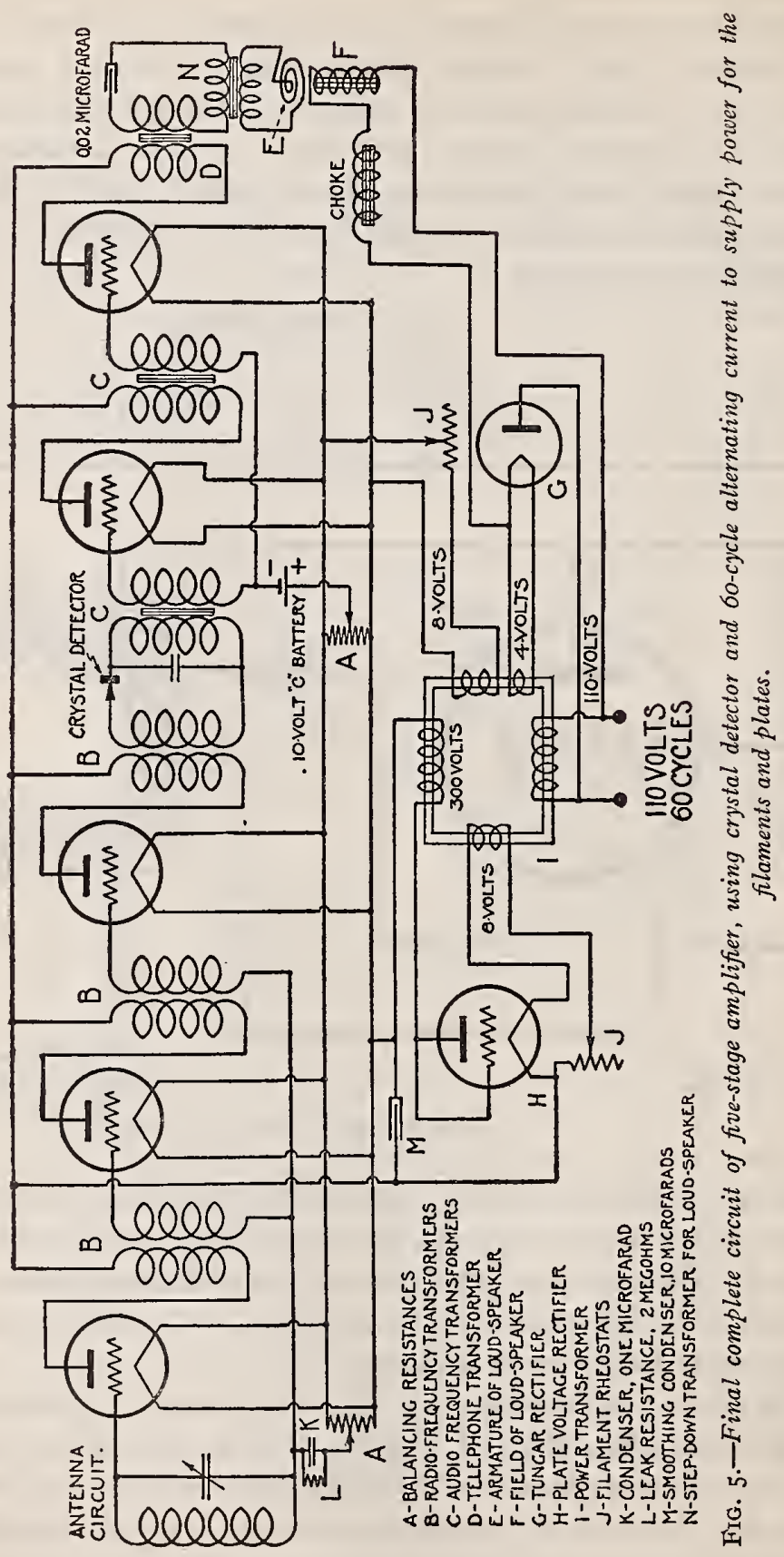


Scientific Papers of the Bureau of Standards, Vol. 18

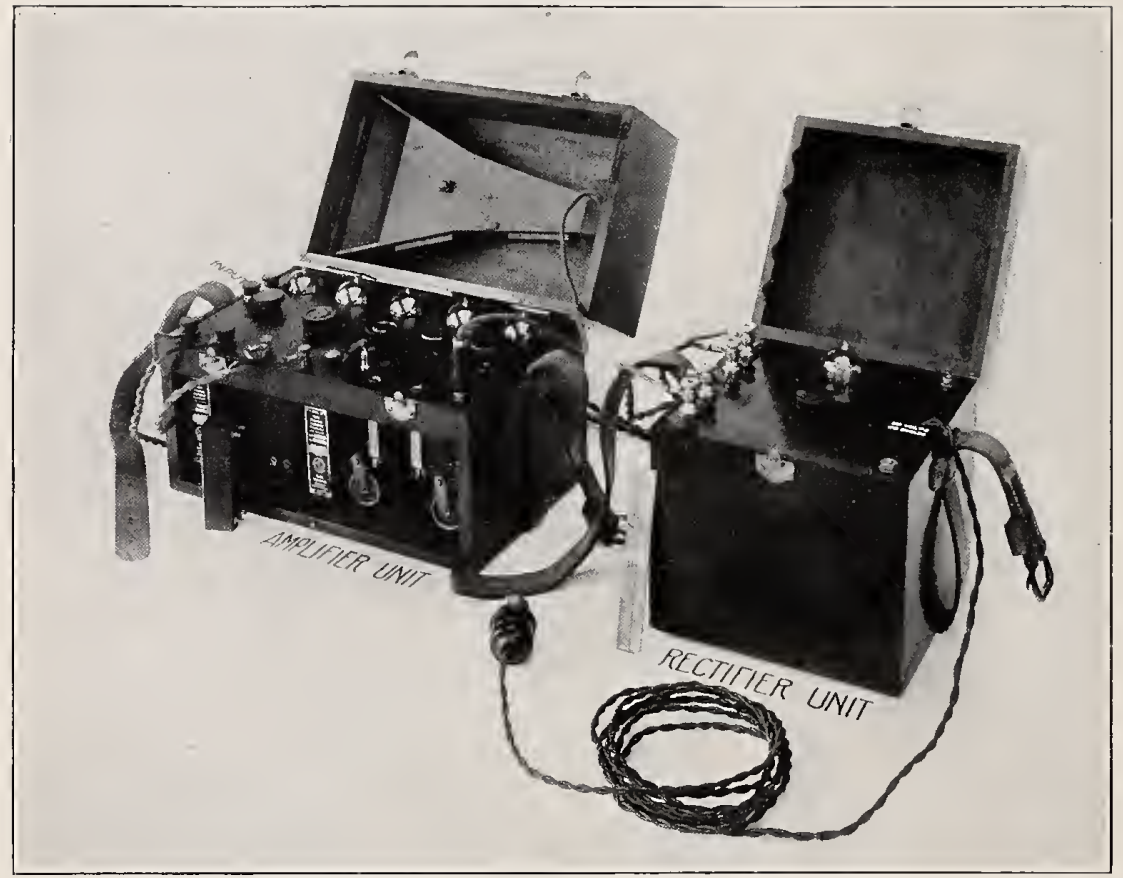

Fig. 6.-Complete assembled amplifier unit and rectifier unit, showing connection for 6o-cycle a-c supply. 
use on the plates of the amplifier tube. Rheostat $Z$ varies the brilliancy of the filament of the rectifier tube and, simultaneously, the voltage for the plates.

The use of a loud-speaking telephone receiver, such as the "Magnavox," was made possible by applying to the field coil of the loud speaker an alternating current rectified by a "Tungar" rectifier tube. The impedance of the field coil was sufficient to smooth out the pulsating current to such an extent that the hum was not annoying. It was also advantageous to couple the loudspeaking reproducer circuit to the plate circuit of the last amplifier tube by means of a one-to-one ratio telephone transformer with a 0.02 microfarad condenser in series with the telephone circuit. This helped considerably to reduce the residual hum in the telephone.

The final circuit is shown in Figure 5, and includes three stages of radio-frequency amplification, galena crystal detector, two stages of audio-frequency amplification, loud-speaking reproducer, and the necessary power transformer and rectification circuits.

This final circuit gives good amplification, with a slight residual hum, which is not great enough to be objectionable, when receiving signals of ordinary readable strength. The residual hum is, of course, more objectionable when extremely weak signals are being received. Radiotelephone music and conversation are clearly reproduced.

The amplifier was operated under normal conditions, using the usual sources of direct-current supply, and then switched over to alternating-current supply. This comparative test showed the alternating-current supply to give as good amplification as the direct-current supply, although the set was slightly more sensitive to weak signals when batteries were used, on account of the interference caused by the hum when used on alternatingcurrent.

For the reception of damped waves, the amplifier as constructed operated most satisfactorily for frequencies from 400 kilocycles to I 500 kilocycles per second ( 750 to 200 meters). This frequency range was determined by the working range of the radio-frequency transformers used. By using suitable radio-frequency transformers, it is expected that the amplifier will be effective for the reception of damped waves for frequencies as low as 30 kilocycles (Io 000 meters). This amplifier has also been found effective for the reception of undamped waves, when used with a separate heterodyne. 
The special transformer with five windings and the rectifier tube were assembled in one box, and the amplifier tubes and amplifier transformers and other apparatus were assembled in a separate box. This was done to avoid having the amplifier immediately adjacent to the special transformer, from which it would pick up considerable 6o-cycle hum. The assembled rectifier unit measured about 8 by 8 by 9 inches and weighed about 21 pounds. The assembled amplifier unit measured about 8 by i i by i 4 inches and weighed about $2 \mathrm{I}$ pounds.

Figure 6 shows the rectifier unit and the amplifier unit as completed, with the plug to connect with the 6o-cycle lighting current. A 6-inch rule is shown leaning against the rectifier unit.

F. W. Dunmore was a coworker and participated in the development of this instrument.

WASHington, June I 7 , 1922. 